\title{
Auditory Brainstem Response Latency in Noise as a Marker of Cochlear Synaptopathy
}

\author{
(1)Golbarg Mehraei, ${ }^{1,2}$ (DAnn E. Hickox, ${ }^{1,4}$-Hari M. Bharadwaj, ${ }^{2,7}$ Hannah Goldberg, ${ }^{1}$ Sarah Verhulst,, ${ }^{2,6}$ \\ M. Charles Liberman, ${ }^{1,4,5}$ and ${ }^{-B}$ Barbara G. Shinn-Cunningham ${ }^{2,3}$ \\ ${ }^{1}$ Program in Speech and Hearing Bioscience and Technology, Harvard University/Massachusetts Institute of Technology, Cambridge, Massachusetts 02139, \\ ${ }^{2}$ Center for Computational Neuroscience and Neural Technology, Boston University, Boston, Massachusetts 02215, ${ }^{3}$ Department of Biomedical \\ Engineering, Boston University, Boston, Massachusetts 02215, ${ }^{4}$ Eaton-Peabody Laboratory, Massachusetts Eye and Ear Infirmary, Boston, Massachusetts \\ 02114, ${ }^{5}$ Department of Otology and Laryngology, Harvard Medical School, Boston, Massachusetts 02114, ${ }^{6}$ Cluster of Excellence Hearing4All and Medical \\ Physics, Department of Medical Physics and Acoustics, Oldenburg University, 26129 Oldenburg, Germany, ${ }^{7}$ Martinos Center for Biomedical Imaging, \\ Department of Neurology, Massachusetts General Hospital/Harvard Medical School, Charlestown, Massachusetts 02129
}

Evidence from animal and human studies suggests that moderate acoustic exposure, causing only transient threshold elevation, can nonetheless cause "hidden hearing loss" that interferes with coding of suprathreshold sound. Such noise exposure destroys synaptic connections between cochlear hair cells and auditory nerve fibers; however, there is no clinical test of this synaptopathy in humans. In animals, synaptopathy reduces the amplitude of auditory brainstem response (ABR) wave-I. Unfortunately, ABR wave-I is difficult to measure in humans, limiting its clinical use. Here, using analogous measurements in humans and mice, we show that the effect of masking noise on the latency of the more robust ABR wave-V mirrors changes in ABR wave-I amplitude. Furthermore, in our human cohort, the effect of noise on wave-V latency predicts perceptual temporal sensitivity. Our results suggest that measures of the effects of noise on $\mathrm{ABR}$ wave-V latency can be used to diagnose cochlear synaptopathy in humans.

Key words: auditory brainstem response; auditory nerve loss; cochlear synaptopathy; hidden hearing loss; temporal coding

\section{Significance Statement}

Although there are suspicions that cochlear synaptopathy affects humans with normal hearing thresholds, no one has yet reported a clinical measure that is a reliable marker of such loss. By combining human and animal data, we demonstrate that the latency of auditory brainstem response wave- $\mathrm{V}$ in noise reflects auditory nerve loss. This is the first study of human listeners with normal hearing thresholds that links individual differences observed in behavior and auditory brainstem response timing to cochlear synaptopathy. These results can guide development of a clinical test to reveal this previously unknown form of noise-induced hearing loss in humans.

\section{Introduction}

Of adult patients seeking audiological help for hearing difficulties, 5-15\% have normal hearing thresholds (NHTs; Kumar et al., 2007; Hind et al., 2011). Such listeners are often diagnosed with

\footnotetext{
Received Dec. 14, 2015; revised Feb. 17, 2016; accepted Feb. 19, 2016.

Author contributions: G.M., A.E.H., H.M.B., S.V., M.C.L., and B.G.S.-C. designed research; G.M., A.E.H., and H.M.G. performed research; G.M. analyzed data; G.M., A.E.H., H.B., S.V., M.C.L., and B.G.S.-C. wrote the paper.

This work was supported by a fellowship from the Office of Assistant Secretary of Defense for Research and Engineering (B.G.S.-C.), National Institute on Deafness and Other Communication Disorders Grant T32 DC-00038 (G.M., A.E.H.), National Institute on Deafness and Other Communication Disorders Grants R01 DC-00188 (M.C.L.) and P30 DC-05029 (M.C.L.), and the Amelia Peabody Charitable Fund (A.E.H.). We thank K. Hancock for assistance in implementing mouse-specific masking noise and L. D. Liberman for expert assistance in cochlear histological processing.

The authors declare that they have no competing financial interests.

Correspondence should be addressed to Golbarg Mehraei, 677 Beacon Street, Boston, MA 02215. E-mail: gmehraei@mit.edu.

DOI:10.1523/JNEUROSCI.4460-15.2016

Copyright $\odot 2016$ the authors $\quad 0270-6474 / 16 / 363755-10 \$ 15.00 / 0$
}

“central auditory processing disorders" (Chermak and Musiek, 1997), suggesting that a normal audiogram means that sounds are robustly represented in auditory nerve fibers (ANFs). However, recent animal studies reveal that noise exposure causing only temporary threshold shifts can produce permanent "cochlear synaptopathy," i.e., loss of synapses and cochlear-nerve terminals innervating inner hair cells (Kujawa and Liberman, 2009; Furman et al., 2013; Liberman and Liberman, 2015). Aging also destroys synaptic connections, independent of hair cell loss (Schmiedt et al., 1996; Makary et al., 2011; Sergeyenko et al., 2013). Still, losses of up to $80 \%$ of synapses do not affect audiometric thresholds in quiet (Lobarinas et al., 2013), rendering such loss invisible to traditional clinical tests.

Models and recent human data suggest that cochlear synaptopathy degrades the coding of temporal cues in suprathreshold sound (Lopez-Poveda and Barrios, 2013; Bharadwaj et al., 2014, 2015). Indeed, in NHT listeners, there are large individual differences in be- 
havioral measures of temporal coding that correlate with physiological brainstem measures affected by ANF loss (Plack et al., 2014; Bharadwaj et al., 2015; Shaheen et al., 2015). Greater noise exposure corresponds to smaller amplitude of the ANF-generated ABR wave-I (Stamper and Johnson, 2015), consistent with the effects of cochlear synaptopathy on ABR wave-I in animals.

ANFs with low-spontaneous discharge rates (low-SR; SR $<20$ spikes/s) are particularly susceptible to neurodegeneration caused by noise and aging (Schmiedt et al., 1996; Furman et al., 2013; Bourien et al., 2014; Liberman and Liberman, 2015). These fibers, which have higher thresholds than high-SR fibers (Liberman, 1978), may be important for hearing in noisy environments, because they are more resistant to masking by background noise (Costalupes, 1985; Young and Barta, 1986). Thus, low-SR synaptopathy could reveal itself through difficulties processing suprathreshold sound, although it has no discernible effect on thresholds.

We hypothesized that variation in low-SR ANF populations underlies the variations in hearing ability among NHT listeners. A test that reveals the fidelity of the peripheral representation of suprathreshold sound and quantifies ANF loss could help diagnose communication disorders in NHT listeners.

Although ABR wave-I amplitude provides an objective measure of ANF loss in animals, it is difficult to measure robustly in the clinic. However, ABR wave-V, generated in the lateral lemniscus and inferior colliculus (Møller and Jannetta, 1985), is robust in humans and can be recorded at low stimulus levels and in background noise. Unfortunately, ABR wave-V amplitude is not reduced by cochlear synaptopathy (Sergeyenko et al., 2013). Here, we concentrate on the shift in ABR wave-V latency in increasing levels of background noise (Burkard and Hecox, 1983, 1987; Burkard and Sims, 2002). This shift could reflect activity of low-SR fibers, because relative to high-SR fibers, they have a delayed onset response (Rhode and Smith, 1985; Bourien et al., 2014) and are more resistant to background noise (Costalupes, 1985; Young and Barta, 1986). Selective loss of low-SR fibers should yield smaller ABR latency shifts as noise level increases. Furthermore, this latency shift should correlate with perceptual measures of fine temporal encoding, which may rely on low-SR responses (Bharadwaj et al., 2015).

In a cohort of young NHT listeners, we measured ABRs in quiet and noise, as well as fine temporal coding precision, i.e., envelope interaural timing difference (ITD) sensitivity. Complementing the human data, masked ABRs were measured in mice with and without noise-induced ANF loss. We find a significant relationship between $A B R$ wave-V latency shifts and the sensitivity to envelope ITD: human subjects with the worst temporal sensitivity showed the smallest slope relating wave-V latency to noise level. Correspondingly, the slope relating ABR wave-IV (analogous to human wave-V) latency to masker level was smaller in mice with noise-induced synaptopathy than in unexposed controls.

\section{Materials and Methods}

\section{Human measurements}

All measures were obtained with the participants seated in an acoustically and electrically shielded booth (single-walled Eckel C-14 booth; Eckel Industries). For passive ABR measures, participants watched a silent, captioned movie of their choice, ignoring the acoustic stimuli. A personal desktop computer controlled all aspects of the experiment, including sound delivery and storing data. Special-purpose sound-control hardware (System 3 real-time signal processing systems, including digital-toanalog conversion and amplification; Tucker Davis Technologies) presented sound through insert phones coupled to foam ear tips. ER-1 insert phones (Etymotic) were used for ABR wave-I and behavioral ITD
Table 1. A summary of the measures and the corresponding stimuli used

\begin{tabular}{|c|c|}
\hline Measure & Stimulus \\
\hline Audiogram & $250 \mathrm{~Hz}$ to $8 \mathrm{kHz}$ pure tones \\
\hline Human CEOAE & $\begin{array}{l}80 \mu \text { s click at levels of } 50-90 \mathrm{~dB} \text { peSPL in } 10 \\
\mathrm{~dB} \text { steps }\end{array}$ \\
\hline Envelope ITD detection & $\begin{array}{l}2 \text { and } 4 \mathrm{kHz} \text { transposed tone in notched noise } \\
\text { modulated at } 50 \mathrm{~Hz} \text { ( } 80 \mathrm{dBSPL})\end{array}$ \\
\hline Human ABR wave-V & $\begin{array}{l}80 \mu \text { s click at levels of } 50-90 \mathrm{~dB} \text { peSPL in } 10 \\
\mathrm{~dB} \text { steps }\end{array}$ \\
\hline Human masked $A B R$ & $\begin{array}{l}80 \mu \text { s click at } 80 \mathrm{~dB} \text { peSPL with broadband } \\
\text { noise varying from } 42 \text { to } 82 \mathrm{~dB} \text { SPL in } 10 \\
\mathrm{~dB} \text { steps }\end{array}$ \\
\hline Human ABR wave-I & $\begin{array}{l}80 \mu \text { s click at levels of } 60-100 \mathrm{~dB} \text { peSPL in } \\
10 \mathrm{~dB} \text { steps }\end{array}$ \\
\hline Mouse masked ABR & $\begin{array}{l}32 \mathrm{kHz} \text { tone pips at } 60 \text { and } 80 \mathrm{~dB} \text { SPL with } \\
\text { broadband noise varying from }-5 \text { to } 85 \\
\mathrm{~dB} \text { SPL in } 5 \mathrm{~dB} \text { steps }\end{array}$ \\
\hline Mouse ABR wave-I & $\begin{array}{l}32 \mathrm{kHz} \text { tone pips at levels of } 15-80 \mathrm{~dB} \text { SPL in } \\
5 \mathrm{~dB} \text { steps }\end{array}$ \\
\hline Mouse DPOAE & $\begin{array}{l}\text { f2 varied from } 8 \text { to } 45.3 \mathrm{kHz} \text { in half-octave } \\
\text { steps; for each f2, L2 level was swept from } \\
10 \text { to } 80 \mathrm{~dB} \text { SPL in } 5 \mathrm{~dB} \text { steps }\end{array}$ \\
\hline
\end{tabular}

measures. ER-10C earphones were used for the ABR in noise measurements to simultaneously record click-evoked otoacoustic emissions (CEOAEs). All sounds were digitized at a sampling rate of $48.828 \mathrm{kHz}$. For the behavioral experiments, subjects responded by button presses. Table 1 summarizes the stimuli used for each experiment.

Participants. Thirty-two (15 females) subjects, aged $20-40$ years, were recruited from the Boston University and Massachusetts Institute of Technology community. Of the 32 subjects, four had uninterpretable ABRs as a result of signal-to-noise issues, equipment, and/or muscle artifact, one listener had tinnitus, two had elevated hearing thresholds, and two were unable to perform the ITD task at above-chance levels. The remaining 23 eligible subjects (nine females; mean age, 26.95 years) had pure-tone hearing thresholds better than $15 \mathrm{~dB}$ hearing level in both ears at octave frequencies between $250 \mathrm{~Hz}$ and $8 \mathrm{kHz}$; all had recorded ABRs in noise and in quiet. Subjects provided informed consent in accordance with protocols established at Boston University. Subject availability precluded some subjects from completing some measures. The number of subjects who completed a task is noted when appropriate.

Correlate of cochlear mechanics. To obtain an objective correlate of cochlear-mechanical compression, CEOAEs were measured simultaneously with the ABR measurements using $80 \mu$ s clicks at levels of 50-90 dB peak equivalent SPL (peSPL) in $10 \mathrm{~dB}$ increments. Each click level was presented 3000 times. CEOAEs were filtered using a 250-6000 Hz bandpass filter because they are most reliable from 1000 to $3000 \mathrm{~Hz}$ (Franklin et al., 1992). To obtain robust estimates despite artifacts, trials were combined by calculating the sample by sample median amplitude instead of the mean. The spectrum of the CEOAEs was computed, and the magnitude was compared with the spectrum of the noise floor, defined as the difference between the odd and even trials.

To provide more frequency-specific information, CEOAE responses were band filtered, in octave bands centered at 1000, 2000, and $4000 \mathrm{~Hz}$, and the signal power for each band was calculated. The CEOAE growth function at each band is defined as the difference between the CEOAEs power (in decibels) and the input level. Across listeners, reliable CEOAEs were obtained at least in one octave-band at click levels $>60 \mathrm{~dB}$ peSPL. As such, the amount of compression in each frequency band, defined as the slope of the CEOAE growth function using a linear fit on log-scale, was determined using CEOAEs at click levels of 70, 80, and $90 \mathrm{~dB}$ peSPL.

Behavioral measure of temporal sensitivity. To obtain a binaural measure of temporal coding, detection of ongoing envelope ITD was measured using a "transposed" tone with a carrier at either 2 or $4 \mathrm{kHz}$ and an envelope modulation frequency of $50 \mathrm{~Hz}$ (Bernstein and Trahiotis, 2002). Figure 1 illustrates the stimuli and the trial setup in this task. The carrier phase was identical in the two ears; the ITD was applied only to the $50 \mathrm{~Hz}$ envelope. The 

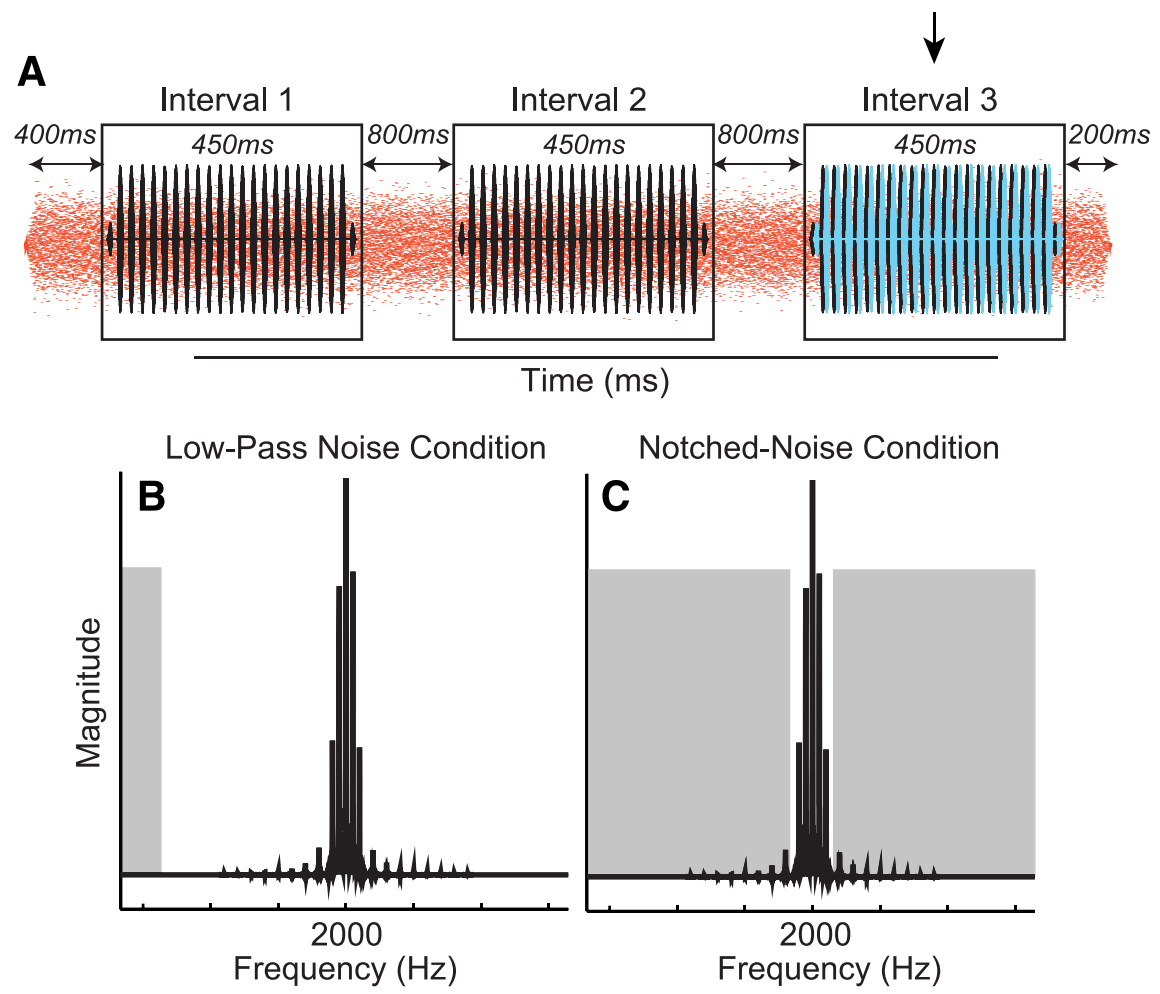

Figure 1. Experiment design of envelope ITD task. $\boldsymbol{A}$, Each trial consists of three intervals. The ITD shift is imposed on the envelope of the transposed tone in either the second or third trial, and the listener is asked to identify this shift. In this example, the ITD shift occurs in the third interval as depicted by the arrow. The background noise turns on $400 \mathrm{~ms}$ before the first interval and halts $200 \mathrm{~ms}$ after the last interval. $\boldsymbol{B}$, The spectrum of the transposed tone presented simultaneously with a low-pass noise. This condition was implemented for the $2 \mathrm{kHz}$ carrier. $\boldsymbol{C}$, Spectrum schematic of transposed tone presented simultaneously with notched noise. This condition was used for both 2 and $4 \mathrm{kHz}$ carriers.

$450 \mathrm{~ms}$ stimulus was ramped with a $20 \mathrm{~ms} \cos ^{2}$ rise decay (simultaneously in the two ears) to minimize the use of onset cues. The stimulus level was set to $80 \mathrm{~dB}$ SPL. Off-frequency notched-noise maskers, realized independently in each trial, were presented at a signal-to-noise ratio of $10 \mathrm{~dB}$ (broadband $\mathrm{rms}$ ) as shown in Figure 1C. The off-frequency masker extended to $20 \mathrm{~Hz}$ on the low-frequency side and $20 \mathrm{kHz}$ on the high-frequency side and served to attenuate off-frequency listening cues, including cues from distortion products. The bandwidth of the notch was set to the equivalent rectangular bandwidth (ERB) of the respective carrier frequency (i.e., ERB at $2 \mathrm{kHz}=240.58$ $\mathrm{Hz}$ and at $4 \mathrm{kHz}=456.46 \mathrm{~Hz}$; Glasberg and Moore, 1990). To determine whether limiting listeners to use of within-channel timing information enhances individual differences in temporal coding, we tested an additional noise condition for the $2 \mathrm{kHz}$ carrier (Fig. $1 B$ ). A diotic noise, low-pass filtered at $1.3 \mathrm{kHz}$, was presented with the stimulus to preclude the listeners' use of any information at low frequencies, such as distortion products (Bernstein and Trahiotis, 2002). Conditions were presented in alternating blocks. All noises were gated on $400 \mathrm{~ms}$ before the first stimulus interval and gated off $200 \mathrm{~ms}$ after the third interval on each trial.

Threshold ITDs were determined using a three-cue, two-alternative forced-choice adaptive task as shown in Figure 1A. Each interval, marked visually by a computer monitor, occurred at intervals of $800 \mathrm{~ms}$. The first interval always contained the stimulus with an ITD of 0 and served as a reference interval. The listener's task was to detect and identify the presence of an ITD (right ear leading) that was presented with equal probability in the second or third interval. A combination of nonparametric, two-up onedown, and parametric, maximum likelihood procedure (MLP) was used to determine the ITD threshold. The two-up one-down procedure (Levitt, 1971) was used initially for seven reversals. The listener was presented with a starting ITD of $900 \mu \mathrm{s}$. The initial step size was $100 \mu \mathrm{s}$. After two reversals, the step size was reduced to $50 \mu \mathrm{s}$ and $25 \mu \mathrm{s}$ after four reversals.

The threshold estimation was then switched to the MLP (Grassi and Soranzo, 2009). In this procedure, a set of psychometric functions with the same slope, attentional lapse rate, and chance level but different midpoints was hypothesized. After each trial, the subject's responses up to that trial were used to calculate the likelihood of each hypothesis (i.e., each psychometric function). The ITD of the next presented trial was chosen based on the $80.9 \%$ correct point on the psychometric function with the highest likelihood. This procedure is repeated until the ITD estimates of the last four trials are within an $80 \mu$ s range of each other.

Each condition was repeated twice for a total of four blocks in each session. Additional blocks were presented if any of the runs for a condition were off by more than 1 SD from the mean. The final ITD threshold was defined as the mean of the thresholds, discarding outliers.

$A B R$ measures. Click-evoked ABRs were measured dichotically using a 32-channel EEG system (Biosemi Active II system). The better ear was chosen for testing based on the subject's audiogram. If there was no difference between the ears audiometrically, the left ear was chosen. ABRs were collected using a $80 \mu$ s click presented at a rate of $10 \mathrm{~Hz}$ via ER-10C insert earphones. A 20 ms interclick jitter was introduced to avoid the accumulation of any stationary interference including the $60 \mathrm{~Hz}$ power-line noise.

ABR wave- $\mathrm{V}$ measures were recorded for five different click levels varying from 50 to 90 $\mathrm{dB}$ peSPL in $10 \mathrm{~dB}$ steps. Masked ABRs were measured using a click presented at $80 \mathrm{~dB}$ peSPL in broadband background noise varying from 42 to $82 \mathrm{~dB}$ SPL in $10 \mathrm{~dB}$ steps. The bandwidth of the noise was limited by the sampling rate used to generate the stimuli and the frequency response of the earphones. Each condition had 3000 repetitions and was presented in randomly ordered blocks. The 32 channels were referenced to the average of the mastoid channels. This measurement took $\sim 1 \mathrm{~h}$ for both the in-quiet and in-noise ABR conditions.

To obtain ABR wave-I, an ER-1 insert earphone electrode was used in addition to the 32 channels. Click levels for the wave-I measurements varied from 60 to $100 \mathrm{~dB}$ peSPL in $10 \mathrm{~dB}$ steps. The polarity of the click stimulus was alternated to prevent the interference of the cochlear microphonic with the ABR. ER-1 electrodes were referenced to the mean of the $\mathrm{Cz}$ and $\mathrm{Fz}$ channels.

Recorded data sampled at $16.384 \mathrm{kHz}$ were pass-band filtered between 100 and $2000 \mathrm{~Hz}$. Power-line noise (60 Hz and harmonics) was removed by applying Thomson's regression method for detecting sinusoids in signals as implemented in the Chronux toolbox (Bokil et al., 2010). The filtered data were then time epoched from -5 to $10 \mathrm{~ms}$ relative to the onset of the stimulus. Bad channels and trials were removed by analyzing the distribution of the overall amplitude across channels and trials. Specifically, a channel or trial was removed if its cumulative amplitude was 2 SDs away from the mean of the distribution. Number of channels and trials were equalized across conditions for each subject. Averaged epoched responses from all good channels were combined to yield the ABR.

The averaged across-channel ABR peak-to-baseline amplitude and peak latency of waves-I and -V, respectively, were identified using visual overlay cursors on a computer screen. We verified our picks by using a denoising method that produced clear wave- $\mathrm{V}$ peaks that preserved the timing of the ABR (de Cheveigné and Simon, 2008). We found that, for those subjects for whom this analysis was performed, the wave- $\mathrm{V}$ latencies derived from the denoised responses agreed with the latencies we found from the raw ABR, even at higher noise levels. The slope of the wave-I amplitude with stimulus level and of wave-V latency shift with noise and with stimulus level was computed by fitting a straight line 
across the conditions in which the waveforms were identifiable. A minimum of three conditions in which the ABR waves-I and -V were clear was required for the linear fits.

Statistical tests. Unless otherwise specified, statistical inference was performed by fitting mixed-effects models to the data and adopting a model comparison approach (Baayen et al., 2008). Fixed-effects terms were included for the various experimental factors, whereas subjectrelated effects were treated as random. To not over-parameterize the random effects, models were compared with and without each term using the Akaike information criterion (Pinheiro and Bates, 2000). All model coefficients and covariance parameters were estimated using restricted maximum likelihood as implemented in the lme4 library in R. An $F$ approximation for the type II scaled Wald statistic was used to make inferences about the fixed effects (Kenward and Roger, 1997): this approximation is more conservative in estimating type I error in contrast to the $\chi^{2}$ approximation and performs well even with complex randomeffects covariance structures (Schaalje et al., 2002). The $p$ values and $F$ statistics based on this approximation are reported.

\section{Animal measurements}

Animal subjects and methods have been described previously in detail by Hickox and Liberman (2014). In brief, $63 \mathrm{CBA} / \mathrm{CaJ}$ male mice (The Jackson Laboratory) were used in this study, divided evenly into three groups: two noise-exposed groups and one control group. Of the 63 animals, 54 ( 18 from each group) were used for physiological testing, and nine (three from each group) were used for histological analysis. Mice were exposed in groups to moderate-level noise at 16-18 weeks of age, and cage mates served as unexposed age- and sex-matched controls. Measures of both cochlear and auditory neural function were recorded to assess degree of recovery from noise exposure. Counts of synaptic ribbons per inner hair cell confirmed noiseinduced loss of cochlear synapses. An additional assay, the masked ABR, was recorded to investigate new physiological indicators of noise-induced cochlear synaptopathy, reported for the first time here. All procedures were approved by the Institutional Animal Care and Use Committee of the Massachusetts Eye and Ear Infirmary.

Noise exposure. Mice were exposed for $2 \mathrm{~h}$ to octave-band noise (8-16 $\mathrm{kHz}$ ) at one of two levels: 100 or $94 \mathrm{~dB}$ SPL. The $100 \mathrm{~dB}$ "neuropathic" exposure, in these mice, causes only transient threshold elevation and extensive cochlear synaptopathy and neural loss in the basal half of the cochlea (Kujawa and Liberman, 2009). The 94 dB "non-neuropathic" exposure was designed to cause similar transient threshold elevation and no ANF loss (Hickox and Liberman, 2014), providing a control group for nonspecific exposure-related effects. Mice were placed, awake and unrestrained, in an acoustically transparent cage within a small reverberant chamber. The noise waveform was generated digitally (fifth-order Butterworth filter), amplified (Crown Power Amplifier D75A; Crown Audio), and presented with a compression driver (JBL model 2446H) through an exponential horn in the roof of the chamber.

Physiology: ABR and distortion product otoacoustic emission. ABRs and distortion product otoacoustic emissions (DPOAEs) were measured in the left ear of each mouse 6-10 weeks after exposure, under ketamine/ xylazine anesthesia (100 and $20 \mathrm{mg} / \mathrm{kg}$, respectively, i.p.). A custom acoustic assembly containing two miniature dynamic speakers and an electret condenser microphone (Knowles FG-23329-P07) was placed within millimeters of the tympanic membrane for delivery of all stimuli and recording of DPOAEs. The assembly was calibrated using a $1 / 4-$ inch condenser microphone (Brüel and Kjær), and each recording session began with in-ear calibrations. Stimulus generation and data acquisition were controlled by custom LabVIEW software on a National Instruments PXI system (16- or 24-bit soundcards).

To assess outer hair cell-based cochlear function, DPOAEs were recorded using primary frequencies $\mathrm{f} 1$ and $\mathrm{f} 2(\mathrm{f} 2 / \mathrm{f} 1=1.2)$, where $\mathrm{f} 2=$ $8-45.3 \mathrm{kHz}$ in half-octave steps. For each set of primaries, levels L1 and $\mathrm{L} 2(\mathrm{~L} 1-\mathrm{L} 2=10 \mathrm{~dB})$ were swept from $\mathrm{L} 2=10-80 \mathrm{~dB}$ SPL in $5 \mathrm{~dB}$ steps. Amplitude of the cubic distortion product $2 \mathrm{f} 1-\mathrm{f} 2$ was extracted from averaged ear canal spectra. DPOAE threshold was interpolated from amplitude input/output functions at 2f1-f2 $=5 \mathrm{~dB}$ SPL.

To assess auditory brainstem function in quiet, ABRs were recorded using $4 \mathrm{~ms}$ tone pips ( $0.5 \mathrm{~ms} \cos ^{2}$ rise-fall, alternating polarity, $\left.40 / \mathrm{s}\right)$ at 32
$\mathrm{kHz}$. Levels were swept from 15 to $80 \mathrm{~dB}$ SPL in $5 \mathrm{~dB}$ steps, and, at each level, average waveforms were generated from 1024 presentations (512 stimulus pairs), amplified $10,000 \times$, bandpass filtered from 0.3 to $3 \mathrm{kHz}$, and stored for offline analysis. Responses were differentially recorded from subdermal needle electrodes at the vertex and ventral edge of left pinna, with ground at the base of the tail. ABR wave-I growth functions were constructed using peak 1 amplitude at each level, defined as the difference between a $1 \mathrm{~ms}$ average of the pre-response baseline and the wave-I peak (with additional high-pass filtering to remove lowfrequency baseline shifts).

Masked ABRs were recorded as for ABRs in quiet, using $32 \mathrm{kHz}$ tonepips at 60 and $80 \mathrm{~dB}$ SPL. Broadband masking noise, extending from 4 to $64 \mathrm{kHz}$, was generated and played with each tone-pip presentation. The magnitude spectrum of the masking noise compensated for the CBA/CaJ mouse audiogram constructed from single-unit ANF thresholds, such that a particular noise level would have a similar masking effect across ANFs of different characteristic frequencies (CFs) regardless of their absolute thresholds. This method was implemented using average ANF threshold data binned according to CF in half-octave bands centered at $4-45.3 \mathrm{kHz}$ in half-octave steps (with linear interpolation between points). Masking noise was swept from -5 to $85 \mathrm{~dB}$ SPL in $5 \mathrm{~dB}$ steps. Wave-IV peak latency was defined through visual analysis by two judges, and wave-IV latency slope was calculated as for human data.

Histology: synaptic ribbon counts. Left inner ears were extracted from three mice for each group (100 dB neuropathic exposure, $94 \mathrm{~dB}$ nonneuropathic exposure, unexposed controls), selected as representative ears based on ABR wave-I amplitude. Ears were extracted after intracardiac perfusion ( $4 \%$ paraformaldehyde) while deeply anesthetized and were postfixed for $2 \mathrm{~h}$ at room temperature. After decalcification in EDTA for 2-3 d, the cochlear spiral was microdissected into six whole-mount pieces that were immunostained with the following: primary antibodies overnight at $37^{\circ} \mathrm{C}$ (mouse anti-CtBP2 at 1:200 from BD Transduction Labs; rabbit antiMyosin VIIa at 1:200, no. 25-6790, from Proteus Biosciences) and secondary antibodies for $1 \mathrm{~h}$ at $37^{\circ} \mathrm{C}$ (biotinylated donkey anti-mouse at 1:200, followed by streptavidin-conjugated Alexa Fluor 568 at 1:1000; Alexa Fluor 647-coupled donkey anti-rabbit at 1:200).

Whole-mount pieces were imaged using confocal microscopy (Leica TCS SP2) at specific cochlear frequency locations that were identified using a reconstruction of the cochlear frequency map across pieces (custom ImageJ plug-in). For a given location, two adjacent regions were captured as $z$-stacks using an oil-immersion $100 \times$ objective ( 1.4 numerical aperture) with $2 \times$ digital zoom and a $z$-step of $0.25 \mu \mathrm{m}$. $Z$-stacks of the inner hair cell base area were analyzed offline as $3 \mathrm{D}$ reconstructions in Amira (Visage Imaging). Individual ribbons (anti-CtBP2 puncta) were quantified and expressed as synaptic ribbons per number of inner hair cells in the stack (anti-Myosin VIIa cytoplasmic label).

\section{Results}

The purpose of this study was twofold: to determine (1) whether noise-induced cochlear synaptopathy in animals influences how ABR wave- $V$ latency shifts with increasing background noise level and (2) whether ABR wave-V latency shifts are related to individual differences in suprathreshold temporal coding in young NHT listeners.

Methods and measures were designed to reveal the contributions of low-SR fibers, shown to be vulnerable to noise exposure (Schmiedt et al., 1996; Furman et al., 2013; Liberman and Liberman, 2015), and may be important for coding in acoustic scenes with competing sound sources (Bharadwaj et al., 2014, 2015). Specifically, we used the following: (1) high sound levels, in which the contribution of low-SR fibers to the overall population response is greater, in contrast to low sound intensities in which high-SR fibers dominate (Liberman, 1978); (2) broadband noise maskers for our ABR measures, to highlight the resistance of low-SR fibers to background noise (Costalupes, 1985; Young and Barta, 1986); and (3) off-frequency maskers for our psychophys- 
A

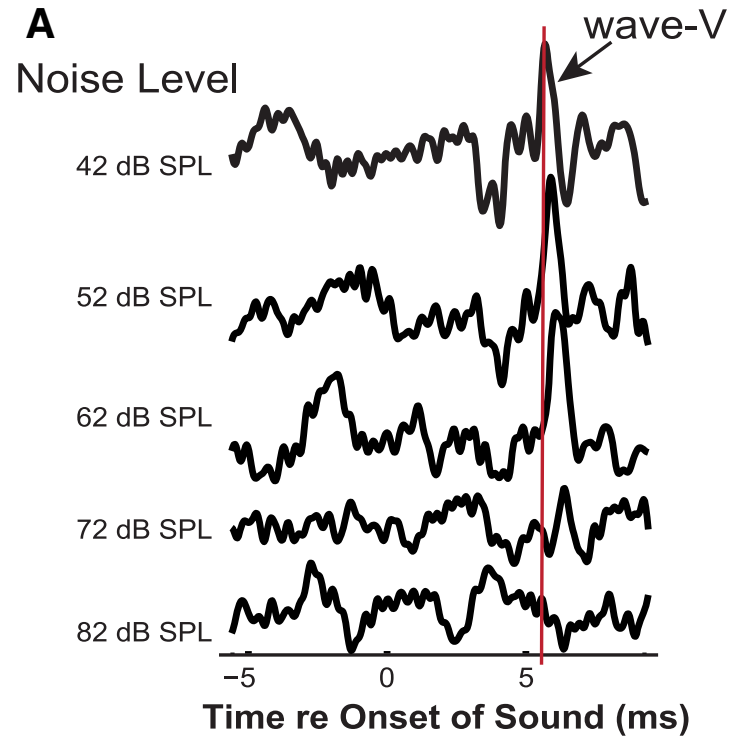

C

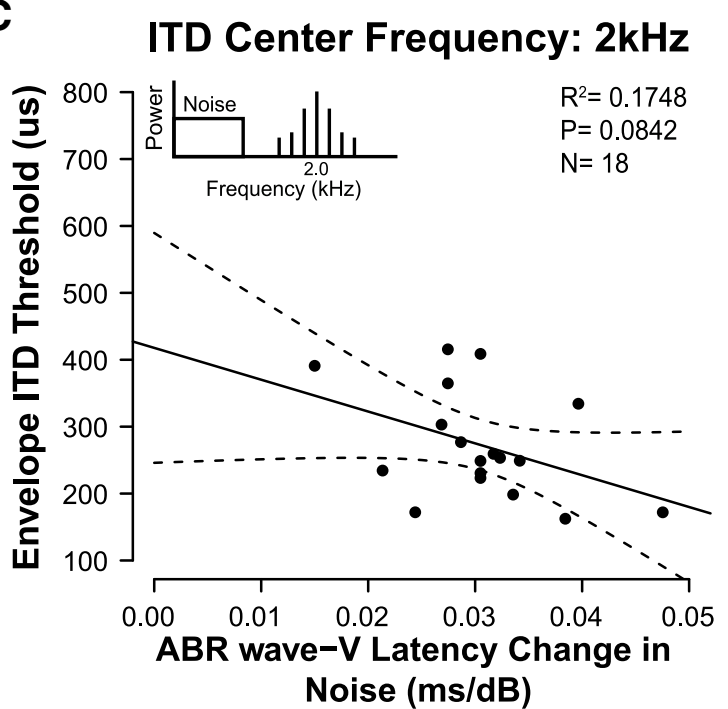

B

ITD Center Frequency: 4kHz

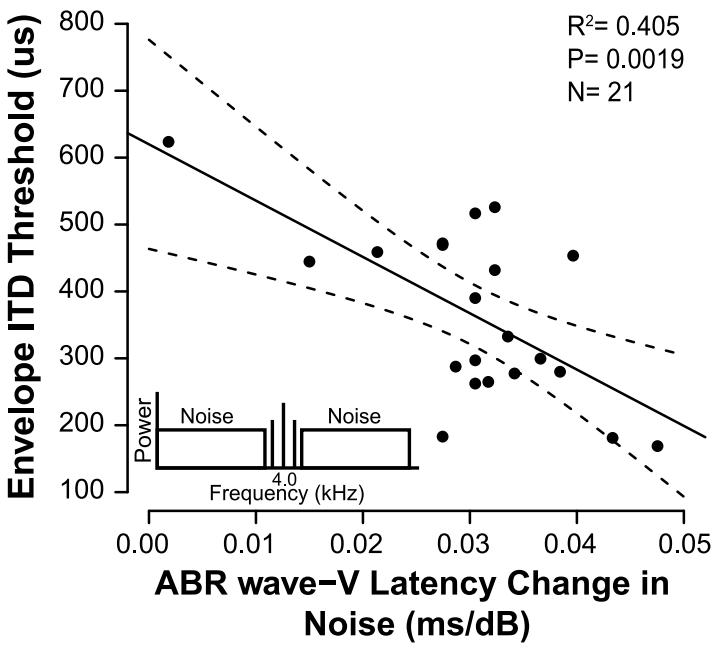

D

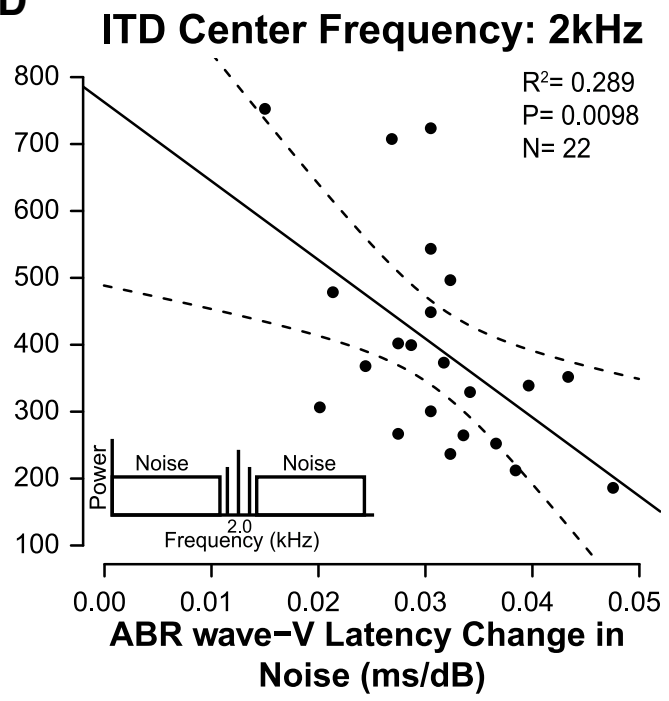

Figure 2. ABR wave-V latency shift in noise correlates with perceptual performance on temporal-cue tasks. A, Sample masked human ABRs using a $80 \mathrm{~dB}$ peSPL click at different background noise levels. Note how wave-V latency shifts with increasing noise level. At each noise level, peak ABR wave-V latency was defined and a linear regression was fitted to all noise level conditions in which ABR wave-V was identifiable for each listener. The slope of each fit was compared with envelope ITD sensitivity measured using a transposed tone with center frequency of $4 \mathrm{kHz}$ ( $\boldsymbol{B})$ and $2 \mathrm{kHz}(\boldsymbol{D})$ presented with notched noise. Notch width was equivalent to the ERB of the used center frequency. $x$-Axis in plots depicts the slope of each fit. There is a significant correlation between sensitivity to envelope ITD and the slope describing ABR wave-V latency shift with noise level when the ITD sensitivity is measured using notched noise. When the transposed tone was presented with a low-pass noise, allowing off-frequency listening ( $(\boldsymbol{C}$, the envelope ITD sensitivity did not correlate with the masked ABR wave-V latency shift. Dashed lines in $\boldsymbol{B}-\boldsymbol{D}$ depict the $90 \%$ confidence interval.

ical measures, to reduce the contribution of off-frequency fibers to encoding of the target stimulus.

Envelope ITD sensitivity was chosen as the target task because sound localization based on timing information relies on the time-locked synchronous response of ANFs (Bernstein and Trahiotis, 2002). We studied sensitivity to envelope ITD with carrier frequencies of 2 and $4 \mathrm{kHz}$ because noise-induced threshold shifts often present as notches $\sim 4 \mathrm{kHz}$, and $\mathrm{ABR}$ wave- $\mathrm{V}$ has contributions from mid to high cochlear frequency regions (Abdala and Folsom, 1995). Additionally, to test whether differences in cochlear amplifier function affect the ABR latency and ITD sensitivity, we measured CEOAEs as a function of click level.

Wave-V latency, wave-I amplitude, and temporal acuity in humans

Click-evoked ABRs recorded with scalp electrodes showed a robust wave-V in both quiet and noise conditions. Responses to stimuli in quiet showed a clear decrease in wave- $\mathrm{V}$ latency with increasing stimulus level (data not shown), consistent with previous reports (Dau, 2003). This latency decrease can be explained by the broadening of peripheral auditory filters with level, yielding shorter impulse responses and an excitation pattern that peaks more basally along the basilar membrane (Neely et al., 1988; Harte et al., 2009). Conversely, masked ABRs showed evidence of increasing wave- $\mathrm{V}$ latency with background noise level (Fig. 2A). Measuring the slope of this latency versus masker-level function, as calculated by a linear fit, helps normalize for intersubject differences in the timing of this wave attributable to gender, age, and head geometry (Mitchell et al., 1989). As seen in Figure $2 B-D$, wave-V latency shift varied over a wide range for our NHT subjects from 0.0018 to 0.0464 $\mathrm{ms} / \mathrm{dB}$. From here on, we will refer to this slope as the "wave-V latency shift." 
Concomitantly, we also found individual differences in the binaural measure of temporal coding fidelity, as observed in previous work (Bharadwaj et al., 2015). As shown in Figure 2, envelope ITD sensitivity for carrier frequencies of 2 and $4 \mathrm{kHz}$ varies widely across our NHT listeners, from $\sim 200$ to $800 \mu$ s when an off-frequency masker was used (Figure $2 B, D$ ). This range is greater, and threshold values are higher, than past work (Dreyer and Oxenham, 2008); these differences may arise because, unlike previous studies, we (1) presented our stimuli at a relatively high intensity, (2) introduced off-frequency maskers, and (3) tested a lower envelope modulation rate. When the stimulus is presented with only a low-pass noise (Fig. $2 C$ ), the envelope ITD thresholds improve and the range of variability decreases, suggesting that listeners benefit from recruitment of off-frequency fibers in this task.

To evaluate the relative contributions of different factors to envelope ITD detection, we used a linear mixed-effects regression model. The model included fixed-effect terms for age, pure-tone thresholds at 2 and $4 \mathrm{kHz}$, gender, carrier frequency, and wave- $\mathrm{V}$ latency shift. Because the 2 and $4 \mathrm{kHz}$ pure-tone thresholds were correlated with envelope ITD threshold, their respective contributions cannot be disentangled from each other. However, because neither is correlated with the wave-V latency shift, including them does not affect inferences about how the wave- $\mathrm{V}$ latency shift is related to envelope ITD coding. The main effect of the wave-V latency shift was significant for predicting the envelope ITD thresholds $\left(F_{(1,14.671)}=15.16, p=0.0015\right)$. Age was not a significant factor; however, this is not surprising given the limited age range of our subjects (all but two were younger than 30 years).

Post hoc analysis revealed that the smaller the wave-V latency shift, the poorer the listener was at detecting changes in envelope ITD when off-frequency listening was limited (Fig. $2 B, D$ ). This correlation was significant for both the $2(r=-0.538, p=$ $0.0098)$ and $4(r=-0.636, p=0.0019) \mathrm{kHz}$ carrier frequencies. However, when off-frequency cues were not masked, the strength of the relationship was reduced and failed to reach significance (two-tailed Pearson's correlation: $r=-0.418, p=0.0842$; Fig. $2 C)$. This is consistent with the interpretation that the individual differences in envelope ITD sensitivity in notched noise (Fig. $2 B, D)$ is driven primarily by the contribution of on-frequency ANFs and may mostly reflect the temporal encoding of low-SR fibers. At higher stimulus levels, as tested here, the low-SR fibers are relatively more synchronized to the envelope modulation of the acoustic signal than high-SR fibers (Bharadwaj et al., 2014). Thus, the timing difference in the envelope of our stimulus may primarily but not entirely be encoded by the low-SR fibers.

To better understand the underlying mechanisms of the wave-V latency shift in noise, we compared these values with the wave-V latency shifts with stimulus level in quiet. The wave- $\mathrm{V}$ latency shift with stimulus level reflects changes attributable to cochlear excitation levels. If this were an important factor affecting ABRs in noise, the latency shift in noise should correlate with the wave-V latency shift in quiet, yet these two measures were unrelated ( $r=-0.3192, p=0.1376)$, suggesting that changes in the cochlear excitation pattern do not cause the wave-V latency shift in noise. Furthermore, no significant relationship was found between wave- $V$ latency shift and how wave- $V$ amplitude changes with either the stimulus level $(r=-0.0059, p=0.9816)$ or the noise level $(r=0.2043, p=0.4016)$. The results are consistent with our hypothesis and suggest that a loss of low-SR fibers yields sound coding deficits that affect both hearing in background noise and in coding temporal information at suprathreshold levels.

Because wave- $\mathrm{V}$ is generated in the brainstem and midbrain (Møller and Jannetta, 1985), it is unclear whether the differences

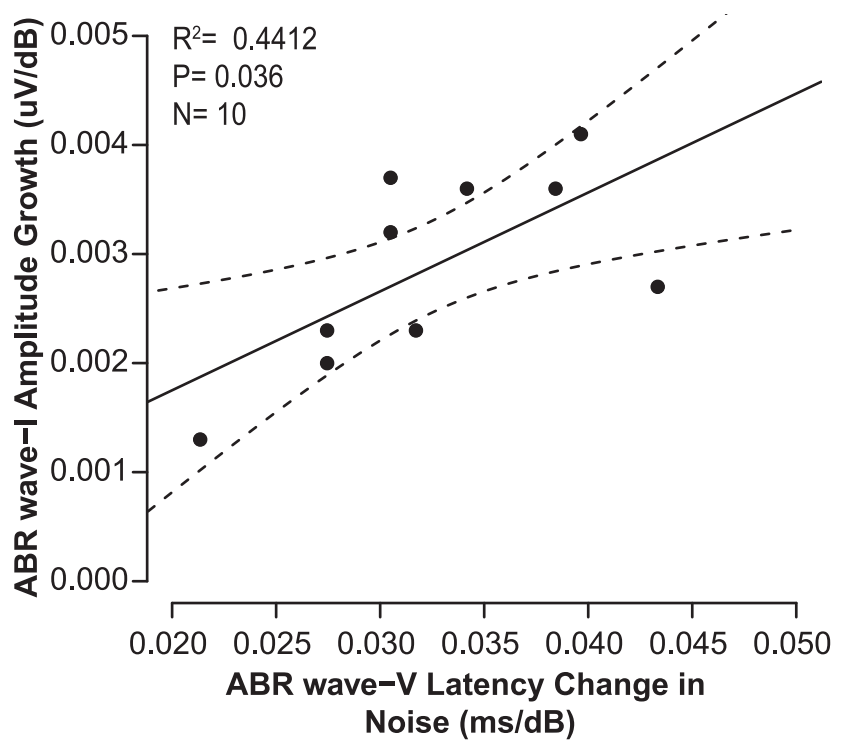

Figure 3. Human ABR wave-I amplitude growth correlates with ABR wave-V latency shift with noise level in $10 \mathrm{NHT}$ listeners. ABR wave-I amplitude was defined by peak amplitude relative to $5 \mathrm{~ms}$ baseline. ABR wave-I amplitude growth slope with click level was calculated using a linear regression. Dashed lines depict the $90 \%$ confidence interval.

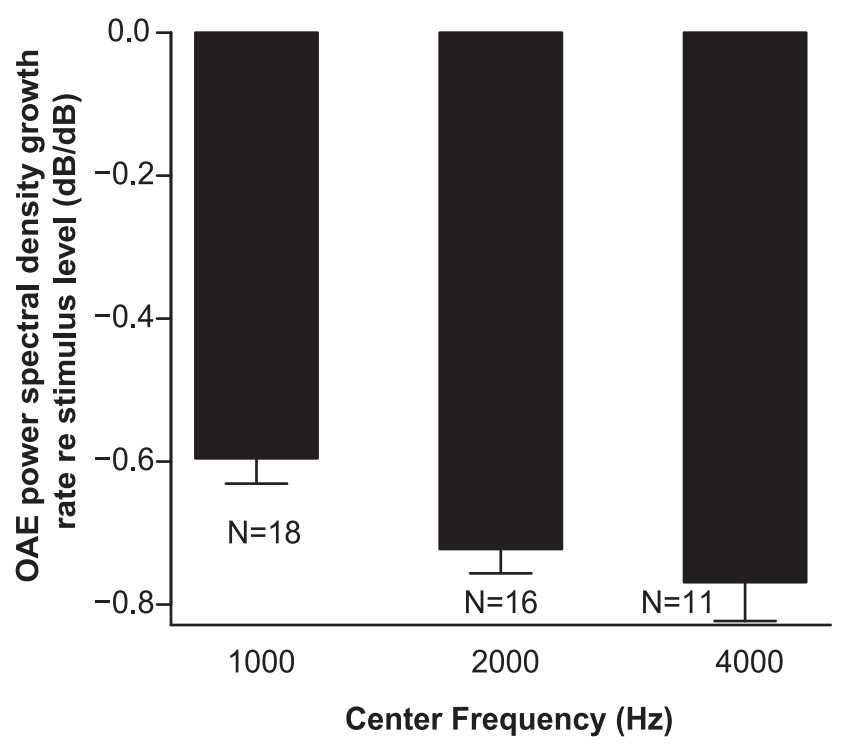

Figure 4. Mean CEOAE spectral power growth rate with increasing stimulus level for each frequency band. Growth rate is the slope of the normalized CEOAE spectral power as a function of the stimulus level. CEOAE spectral power is normalized to the stimulus level. The growth function was fitted on a log scale; thus, a slope of 0 would mean that the CEOAE spectral power grew linearly with increasing stimulus level, and a negative slope depicts a compressive growth. Error bars depict standard error. Notice that the number of subjects varies for each frequency band because not all subjects had reliable CEOAEs in each frequency band.

in the latency shifts are a direct consequence of differences in auditory nerve response or from a later stage of processing. To investigate this, in a subset of our subjects $(n=10)$, we measured ABR wave-I to high-level clicks (60-100 dB peSPL) using earcanal electrodes ("tiptrodes"), positioned closer to the auditory nerve generators. As shown in Figure 3, there was a significant relationship between wave-I growth and wave-V latency shift $(r=0.66, p=0.036)$ : listeners with a steeper wave-I growth curve had larger wave-V latency shifts. This is consistent with our hy- 
Table 2. Correlations for CEOAE versus ITD sensitivity in notched noise and ABR wave-V latency shift in noise

\begin{tabular}{lllr}
\hline $\begin{array}{lll}\text { CEOAE } \\
\text { growth }\end{array}$ & ABR wave-V latency shift & 2 kHz ITD sensitivity & 4 kHz ITD sensitivity \\
\hline $1 \mathrm{kHz}$ & $r=-0.1712, p=0.4971$ & & \\
$2 \mathrm{kHz}$ & $r=0.0064, p=0.9813$ & $r=-0.0025, p=0.9929$ & \\
$4 \mathrm{kHz}$ & $r=-0.4252, p=0.1924$ & & $r=-0.1273$, \\
& & & $p=0.7441$ \\
\hline
\end{tabular}

A
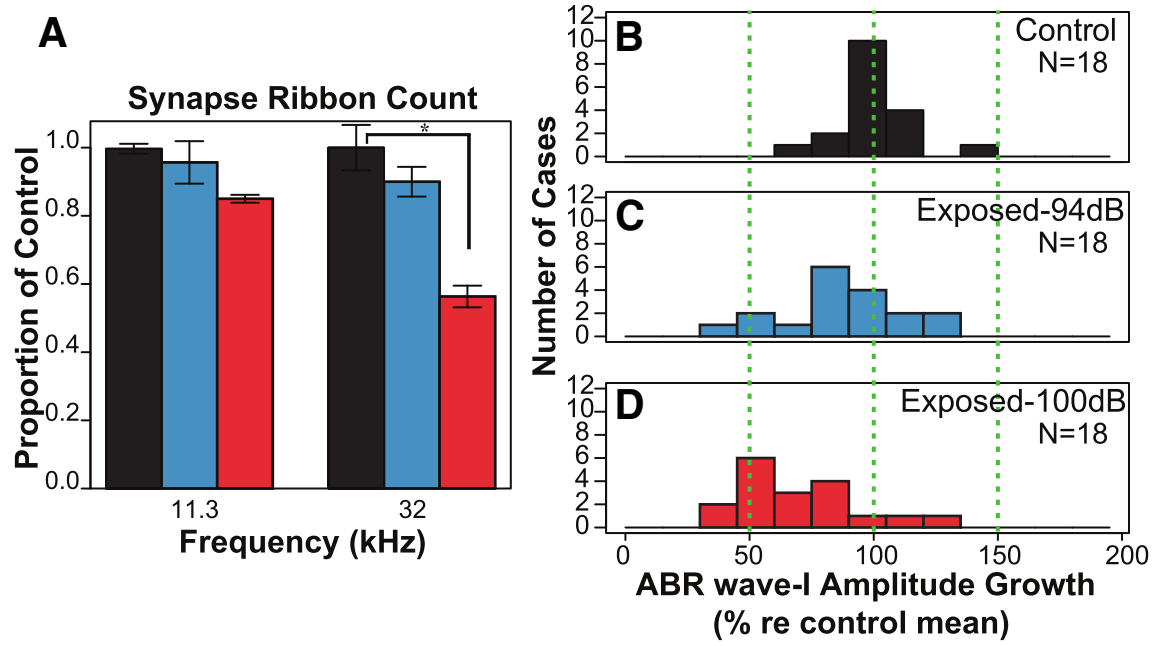

Figure 5. Reduced synaptic ribbon counts and ABR wave-I amplitude growth is observed in mice after neuropathic noise exposure. $\boldsymbol{A}$, Mean presynaptic ribbon counts per inner hair cell across exposure groups, expressed as proportion of unexposed control means. The Exposed-100 dB group (red) showed a significant reduction $\left({ }^{*} p<0.01\right)$ in ribbon counts at the $32 \mathrm{kHz}$ cochlear frequency location relative to the other groups. Error bars depict standard error. Histograms of ABR wave-I amplitude growth with stimulus level for Control (B), Exposed-94 dB (C; non-neuropathic) and Exposed-100 dB ( $\boldsymbol{D}$; neuropathic) groups. Abscissa depicts percentage of wave-I growth relative to the control mean.

pothesis that differences in latency shift arise from changes in the ANF response. In contrast, there was no correlation between wave-I amplitude growth and wave- $\mathrm{V}$ amplitude growth in quiet $(r=-0.1801, p=0.5962)$, in agreement with the idea that, after cochlear damage, there is compensatory gain in the central auditory pathway that maintains wave- $\mathrm{V}$ amplitude (Schaette and McAlpine, 2011; Sergeyenko et al., 2013).

\section{Cochlear amplifier function}

Figure 4 shows the mean CEOAEs spectral power growth rate in each frequency band. The growth rate, defined as the slope of the CEOAEs spectral power, is a measure of cochlear amplifier function linked to the outer hair cells (Shera, 2004). Abnormal cochlear mechanics would result in a more linear than compressive CEOAE growth with click level. Although there are some individual differences depicted by the standard error, we found no significant correlation between these differences and that of the envelope ITD thresholds or the wave- $V$ latency shifts as summarized in Table 2. Thus, the differences we observe in the ABR and ITD measures are not significantly affected by changes in cochlear mechanics, as expected if the underlying cause is related to cochlear synaptopathy.

Wave-IV latency, wave-I amplitude, and cochlear synaptopathy in mice

Although our human data suggest that individual differences in the wave-V latency shift in NHT listeners arise from changes in the auditory nerve response, studying masked ABRs in mice with noise-induced cochlear synaptopathy allows a more direct test of the hypothesis. Sixty-three mice were divided evenly into three groups: (1) unexposed (controls); (2) exposed to octave-band noise $(8-16 \mathrm{kHz})$ at $94 \mathrm{~dB}$ SPL designed to cause only transient threshold elevation and no ANF loss (non-neuropathic); and (3) exposed to the same noise at $100 \mathrm{~dB}$ SPL, causing transient threshold elevation, and no hair cell loss, yet marked degeneration of auditory nerve synapses (neuropathic). Cochlear synaptopathy was assessed in representative ears by immunostaining with an antibody to a major protein in the presynaptic ribbon, present at each synaptic contact between an ANF and the hair cell it contacts (Hickox and Liberman, 2014). The mean ribbon count per hair cell was 15.95, 15.24 , and 13.55 at the $11.3 \mathrm{kHz}$ location and $16.48,14.79$, and 9.22 at $32 \mathrm{kHz}$ for the control, non-neuropathic, and neuropathic groups, respectively. The neuropathic group showed a significant reduction in ribbons per hair cell compared with unexposed controls (44\%) and with the non-neuropathic mice at 32 $\mathrm{kHz}$, whereas the non-neuropathic group showed ribbon counts statistically indistinguishable from control (Fig. 5A). Previous work has shown that the loss of synaptic connections is associated with a corresponding decrease (36\%) in the suprathreshold amplitude of ABR wave-I (Hickox and Liberman, 2014). The wave-I amplitude growth was further reduced in the neuropathic group by $50 \%$ relative to the control, whereas the nonneuropathic mice showed a reduction that was statistically indistinguishable from control (Fig. 5B).

To evaluate ABR latency shifts in noise, we examined wave-IV in mice because it is robust (Hickox and Liberman, 2014) and because wave-I to wave-IV in animals are approximately equivalent to wave-I to wave- $\mathrm{V}$, respectively, in humans (Møller and Jannetta, 1985; Markand, 1994). As with the human data, the ABR wave-IV latency in mice increased with noise level. The mean slope of the latency versus noise level functions was smaller in the neuropathic group relative to control and nonneuropathic animals (Fig. 6). A linear model with group and probe level as predictors yielded significant main effects of group $\left(F_{(2,51)}=4.2, p=0.02\right)$ and probe level $\left(F_{(1,51)}=12.09, p=\right.$ $0.001)$ on wave-IV latency shift with noise level. Post hoc onetailed $t$ tests revealed significant differences between the neuropathic and control mice at both probe levels $(60 \mathrm{~dB}, p=0.0045$; $80 \mathrm{~dB}, p=0.0365)$. There was also a significant difference between the non-neuropathic and neuropathic groups at $80 \mathrm{~dB}$ SPL $(p=0.0328)$. In contrast, no significant differences were found in the wave-IV latency shift between the control and nonneuropathic groups at either probe level (Fig. 6A,B).

To evaluate outer hair cell function, we measured DPOAEs. Mean thresholds were similar between the control ears and the neuropathic group, with a hint of threshold elevation at the highest test frequency (Fig. 7), suggesting mild outer hair cell damage. To examine whether the differences in ABR latency shift are ascribable to slight differences in cochlear thresholds, we designed a linear model including group, probe level, DPOAE thresholds at $32 \mathrm{kHz}$ (where ABRs were measured), DPOAE thresholds at 45 
$\mathrm{kHz}$, and their interactions as predictor variables of wave-IV latency shift. This model revealed only main effects of probe level $\left(F_{(1,48)}=12.14, p=0.0011\right)$ and group $\left(F_{(2,48)}=3.26, p=0.04\right)$; the DPOAE thresholds were not a significant predictor $\left(F_{(1,48)}=0.49, p=0.58\right)$. These results support the idea that the differences between the neuropathic and control mice wave-IV latency shift arise from the loss of ANFs rather than changes in cochlear mechanics.

Similar to the human data, wave-IV latency shifts were correlated with wave-I amplitude growth. To compare the wave-I amplitude growth and wave-IV latency shift, we pooled across all animals $(n=54)$. The animals form a continuum of noise-exposure phenotypes (Fig. $5 B$ ) without large differences in thresholds (Fig. 7), similar to the human subjects in the current study who all had thresholds within the "normal" audiometric range $(<15 \mathrm{~dB}$ hearing level). Using a mixed-effects linear model with wave-I amplitude growth, probe level, DPOAE thresholds at both 32 and $45 \mathrm{kHz}$, and their interactions, we find significant main effects of wave-I amplitude growth $\left(F_{(1,50)}=5.187, p=0.027\right)$ and probe level $\left(F_{(1,50)}=11.75, p=0.0011\right)$. This analysis further supports the idea that cochlear synaptopathy, reflected in ABR wave-I amplitude growth, underlies the ABR wave-IV latency shift differences between the groups of mice.

\section{Discussion}

Recent animal studies have shown that both moderate noise exposure and aging can produce significant degeneration of the synaptic connections between ANFs and hair cells without changes to the hair cells themselves (Kujawa and Liberman, 2009; Makary et al., 2011; Furman et al., 2013; Sergeyenko et al., 2013). This synaptopathy, which can occur immediately after a noise exposure, is followed by a slow degeneration of the cell body and central axon. However, once the synapse is lost, each ANF is silent whether or not its cell body survives. This diffuse neural loss likely degrades aspects of suprathreshold auditory processing, because it appears to be selective for the low-SR ANFs (Furman et al., 2013; Liberman and Liberman, 2015), but it does not elevate thresholds as measured by conventional audiometry. Given these characteristics, this damage has been called "hidden hearing loss"(Schaette and McAlpine, 2011).

Motivated by the animal studies, we and others have looked for correlates of hidden hearing loss in humans. A growing number of studies suggest that intersubject differences in humans with NHTs measured both behaviorally and electrophysiologically can be explained by ANF loss (Bharadwaj et al., 2015; Stamper and Johnson, 2015). Here, we showed that, compared with those with small wave-V latency shifts, NHT listeners showing large wave-V latency shifts with increasing masker level perform better on a sound localization task that requires discrimination of ITDs in sound envelopes. In mice, we showed that cochlear synaptopathy reduced an analogous measure of wave $-\mathrm{V}$ latency shift in noise. In both mice and humans, the maskingnoise latency shift correlates with the growth of ABR wave-I amplitude with stimulus level but not with measures of cochlear mechanics. Together, the data suggest that the latency shift in

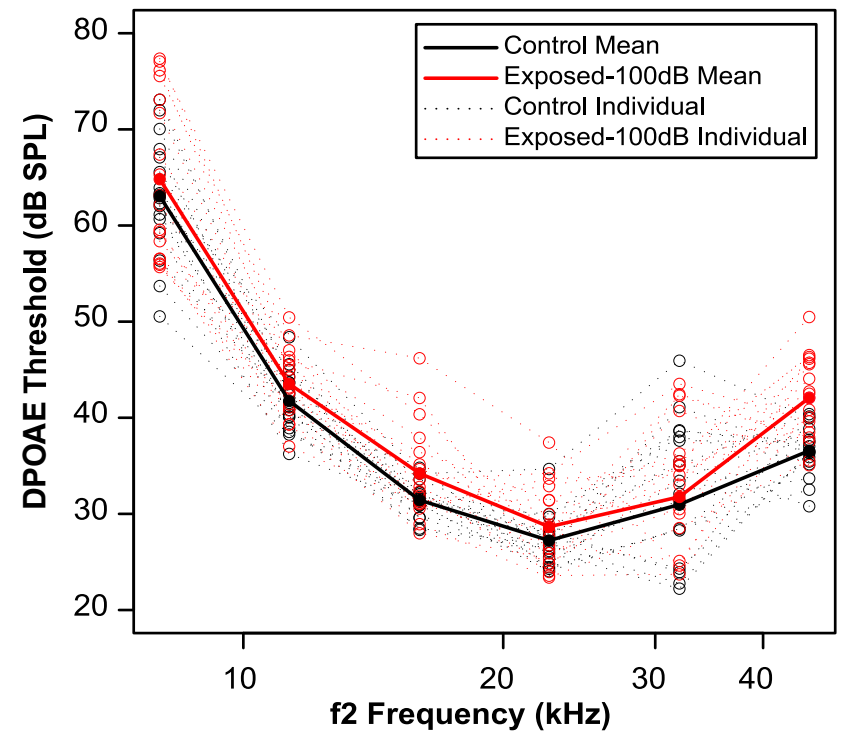

Figure 7. Like the range of thresholds encountered in the NHT listener subject group ( $<15$ $\mathrm{dB}$ hearing level), noise-exposed mice with cochlear synaptopathy show cochlear thresholds within a normal-hearing range (within $15 \mathrm{~dB}$ of controls). Mice exposed to neuropathic (100 dB) noise (red) showed a modest mean elevation in cochlear thresholds in the $45 \mathrm{kHz}$ region compared with unexposed controls (black). Analysis with a statistical linear model suggests that noise-induced differences in DPOAE thresholds, and thus cochlear mechanics, do not account for ABR wave-IV latency shifts with background noise level (see Results). Data for individual mice are shown with dotted lines and open symbols; data for group means are shown with solid lines and filled symbols.

masked ABRs could be a useful metric of hidden hearing loss in humans.

Although we do not demonstrate a preferential loss of low-SR fibers in our mice, there is evidence that low-SR ANFs are more susceptible to noise exposure and aging. In aged gerbils, low- and med-SR fibers in the high CF regions declined from 57 to $29 \%$ of the population (Schmiedt et al., 1996). Similarly, after a $2 \mathrm{~h}$ exposure, Furman et al. (2013) observed low-SR population drop from 47 to $29 \%$ in guinea pigs (Furman et al., 2013). Therefore, we interpret the reduction in the wave-IV latency shift in our neuropathic mice as a result of loss of low-SR fibers.

It is difficult to compare our human results with previous studies because (1) until recently, effects typically have been in- 
vestigated on a group rather than individual level and (2) there has been more focus on absolute rather than relative measures. Nonetheless, evidence of a reduced latency shift in humans has been seen previously in older NHT human listeners (Burkard and Sims, 2002); however, this effect was only seen when the older listeners were divided into "better" and "worse" listeners based on audiometric thresholds and was quite small. Generally, there is a delayed ABR wave-V latency with age (Burkard and Sims, 2002). This is in contrast to what we observed in the couple of listeners we tested who were over the age of 35 years: their absolute masked $\mathrm{ABR}$ latencies were shorter relative to the ABR latencies in our other listeners, who were all younger than the age of 30 years (data not shown). Furthermore, little difference was found in the ABR wave-V latency in quiet at the tested click levels.

The results here corroborate previous findings that implicate a neural rather than cochlear place mechanism underlying wave- $\mathrm{V}$ latency shifts in masked ABRs. Using a high-pass subtractive masking technique and varying broadband noise level, Burkard and Hecox (1987) showed that wave-V latency shifts were greater than could be explained on the basis of shifts in cochlear region of excitation (Burkard and Hecox, 1987). Additionally, there is an interaction between stimulus repetition rate and noise level on wave-V latency (Burkard and Hecox, 1983, 1987). This interaction results in a decrease in rate-induced ABR peak latency shift in the presence of masking noise, suggesting an overlap in the synaptic/neural mechanisms underlying masking-induced and rate-induced latency shifts.

Studies suggest that the ABR wave-V latency shift with noise level is linked to neural desynchronization, originating from either presynaptic events involving the synaptic vesicle cycle or decreased probability of discharge in postsynaptic events. Building on this neural hypothesis, we note that the degree to which noise desynchronizes ANFs is greater in high-SR than low-SR fibers because high-SR fibers have a low response threshold and smaller dynamic range (Liberman, 1978; Rhode et al., 1978). In contrast, low-SR fibers, with their higher thresholds, are more resistant to masking by background noise (Costalupes, 1985; Young and Barta, 1986), and, as such, their relative contribution to the total neural responses increases as noise level increases.

Although cochlear synaptopathy likely affects ABR wave-V latency shift with noise level, alterations in brainstem/central auditory activity may also influence the ABR latency. Hyperactivity in central pathways is seen in mice with synaptic loss induced by noise exposure (Hickox and Liberman, 2014) and may explain why ABR wave-V/I amplitude ratio is increased in tinnitus patients with normal audiograms in which hidden synaptic loss putatively induced by previous noise exposure may also be present (Schaette and McAlpine, 2011). In the inferior colliculus, older mice have a smaller response latency to amplitudemodulated sounds, perhaps attributable to a decrease in inhibition strength with aging (Simon et al., 2004). Furthermore, after carboplatin administration, the absolute wave- $\mathrm{V}$ latency increases, without any change in slope of the latency versus noiselevel curve (Burkard et al., 1997). Carboplatin causes selective inner hair cell loss at low doses. The loss of inner hair cells reduces the strength of both low- and high-SR ANF input to higher auditory centers. Correspondingly, differences in the slope of the wave-V latency versus noise level were not observed in the study by Burkard et al. (1997). Nevertheless, this reduced input may not only lead to delayed responses but also degraded coding of temporal information (Lopez-Poveda and Barrios, 2013). Thus, it is unclear whether and how central changes affect the ABR wave-V latency shift with noise level and how those effects are related to cochlear synaptopathy; more systematic investigation is necessary.

To disambiguate the effects of central versus peripheral changes on later waves of the ABR, we investigated the wave-IV latency shift with noise level in mice with two types of noise exposure. Both caused significant temporary threshold shifts; neither caused significant loss of sensory cells; and only one caused significant loss of ANFs (Hickox and Liberman, 2014). Consistent with our hypothesis, only the neuropathic exposure led to a significant reduction in the wave-IV shift. These results provide empirical evidence that the reduction in wave-IV latency shift with noise level after acoustic injury is due to the loss of ANFs rather than a direct effect of noise exposure on the central auditory system.

We conclude here that differences among NHT listeners, both perceptual abilities and wave-V latency shift with noise level, reflect different degrees of ANF loss. Unlike absolute ABR peak amplitude and latency analyses, in which intersubject variability is large and dependent on numerous factors independent from coding fidelity, we show that the relative change in latency in noise is a robust measure of hidden hearing loss. These testing conditions stress good temporal coding of sound that is clearly audible, mimicking some of the challenges experienced in everyday settings, in which many middle-aged listeners report communication difficulties. It is important to note that this ABR measure should be coupled with a measure of cochlear function, because we do not yet know how any cochlear dysfunction affects ABR wave-V latency in noise. Future studies should investigate the precision of this measure in noise-exposed human cohorts and in listeners with cochlear dysfunction.

\section{References}

Abdala C, Folsom RC (1995) The development of frequency resolution in humans as revealed by the auditory brainstem response recorded with notched noise masking. J Acoust Soc Am 98:921-930. CrossRef Medline

Baayen RH, Davidson DJ, Bates DM (2008) Mixed-effects modeling with crossed random effects for subjects and items. J Mem Lang 59:390-412. CrossRef

Bernstein LR, Trahiotis C (2002) Enhancing sensitivity to interaural delays at high frequencies by using "transposed stimuli." J Acoust Soc Am 112: 1026-1036. CrossRef Medline

Bharadwaj HM, Verhulst S, Shaheen L, Liberman MC, Shinn-Cunningham BG (2014) Cochlear neuropathy and the coding of supra-threshold sound. Front Syst Neurosci 8:26. CrossRef Medline

Bharadwaj HM, Masud S, Mehraei G, Verhulst S, Shinn-Cunningham BG (2015) Individual differences reveal correlates of hidden hearing deficits. J Neurosci 35:2161-2172. CrossRef Medline

Bokil H, Andrews P, Kulkarni JE, Mehta S, Mitra PP (2010) Chronux: a platform for analyzing neural signals. J Neurosci Methods 192:146-151. CrossRef Medline

Bourien J, Tang Y, Batrel C, Huet A, Lenoir M, Ladrech S, Desmadryl G, Nouvian R, Puel JL, Wang J (2014) Contribution of auditory nerve fibers to compound action potential of the auditory nerve. J Neurophysiol 112:1025-1039. CrossRef Medline

Burkard R, Hecox K (1983) The effect of broadband noise on the human brain-stem evoked response. I. Rate and intensity effects. J Acoust Soc Am 74:1204-1213. CrossRef Medline

Burkard R, Hecox KE (1987) The effect of broadband noise on the human brain-stem auditory evoked response. III. Anatomic locus. J Acoust Soc Am 81:1050-1063. CrossRef Medline

Burkard RF, Sims D (2002) A comparison of the effects of broadband masking noise on the auditory brainstem response in young and older adults. Am J Audiol 11:13-22. CrossRef Medline

Burkard R, Trautwein P, Salvi R (1997) The effects of click level, click rate, and level of background masking noise on the inferior colliculus potential (ICP) in the normal and carboplatin-treated chinchilla. J Acoust Soc Am 102:3620-3627. CrossRef Medline 
Chermak GD, Musiek FE (1997) Central auditory processing disorders: new perspectives. San Diego: Singular Publishing Group.

Costalupes JA (1985) Representation of tones in noise in the responses of auditory nerve fibers in cats. I. Comparison with detection thresholds. J Neurosci 5:3261-3269. Medline

Dau T (2003) The importance of cochlear processing for the formation of auditory brainstem and frequency following responses. J Acoust Soc Am 113:936-950. CrossRef Medline

de Cheveigné A, Simon JZ (2008) Denoising based on spatial filtering. J Neurosci Methods 171:331-339. CrossRef Medline

Dreyer AA, Oxenham AJ (2008) Effects of level and background noise on interaural time difference discrimination for transposed stimuli. J Acoust Soc Am 123:EL1-EL7. CrossRef Medline

Franklin DJ, McCoy MJ, Martin GK, Lonsbury-Martin BL (1992) Test/retest reliability of distortion product and transiently evoked otoacoustic emissions. Ear Hear 13:417-429. CrossRef Medline

Furman AC, Kujawa SG, Liberman MC (2013) Noise-induced cochlear neuropathy is selective for fibers with low spontaneous rates. J Neurophysiol 110:577-586. CrossRef Medline

Glasberg BR, Moore BC (1990) Derivation of auditory filter shapes from notched-noise data. Hear Res 47:103-138. CrossRef Medline

Grassi M, Soranzo A (2009) MLP: a MATLAB toolbox for rapid and reliable auditory threshold estimations. Behav Res Methods 41:20-28. CrossRef Medline

Harte JM, Pigasse G, Dau T (2009) Comparison of cochlear delay estimates using otoacoustic emissions and auditory brainstem responses. J Acoust Soc Am 126:1291-1301. CrossRef Medline

Hickox AE, Liberman MC (2014) Is noise-induced cochlear neuropathy key to the generation of hyperacusis or tinnitus? J Neurophysiol 111:552-564. CrossRef Medline

Hind SE, Haines-Bazrafshan R, Benton CL, Brassington W, Towle B, Moore DR (2011) Prevalence of clinical referrals having hearing thresholds within normal limits. Int J Audiol 50:708-716. CrossRef Medline

Kenward MG, Roger JH (1997) Small sample inference for fixed effects from restricted maximum likelihood. Biometrics 53:983-997. CrossRef Medline

Kujawa SG, Liberman MC (2009) Adding insult to injury: Cochlear nerve degeneration after "temporary" noise-induced hearing loss. J Neurosci 29:14077-14085. CrossRef Medline

Kumar G, Amen F, Roy D (2007) Normal hearing tests: is a further appointment really necessary? J R Soc Med 100:66. CrossRef Medline

Levitt H (1971) Transformed up-down methods in psychoacoustics. J Acoust Soc Am 49:Suppl 2:467+. CrossRef Medline

Liberman MC (1978) Auditory-nerve response from cats raised in a lownoise chamber. J Acoust Soc Am 63:442-455. CrossRef Medline

Liberman LD, Suzuki J, Liberman MC (2015) Dynamics of cochlear synaptopathy after acoustic overexposure. J Assoc Res Otolaryngol 16:205-219. CrossRef Medline

Lobarinas E, Salvi R, Ding D (2013) Insensitivity of the audiogram to carboplatin induced inner hair cell loss in chinchillas. Hear Res 302:113-120. CrossRef Medline

Lopez-Poveda EA, Barrios P (2013) Perception of stochastically under- sampled sound waveforms: a model of auditory deafferentation. Front Neurosci 7:124. CrossRef Medline

Makary CA, Shin J, Kujawa SG, Liberman MC, Merchant SN (2011) Agerelated primary cochlear neuronal degeneration in human temporal bones. J Assoc Res Otolaryngol 12:711-717. CrossRef Medline

Markand ON (1994) Brainstem auditory evoked potentials. J Clin Neurophysiol 11:319-342. CrossRef Medline

Mitchell C, Phillips DS, Trune DR (1989) Variables affecting the auditory brainstem response: audiogram, age, gender and head size. Hear Res 40: 75-85. CrossRef Medline

Møller AR, Jannetta PJ (1985) Neural generators of the auditory brainstem response. In: The auditory brainstem response (Jacobson JT, ed), pp 1331. San Diego: College Hill.

Neely ST, Norton SJ, Gorga MP, Jesteadt W (1988) Latency of auditory brain-stem responses and otoacoustic emissions using tone-burst stimuli. J Acoust Soc Am 83:652-656. CrossRef Medline

Pinheiro J, Bates D (2000) Mixed-effects models in S and S-PLUS. New York: Springer.

Plack C, Baker D, Prendergast G (2014) Perceptual consequences of "hidden" hearing loss. Trends Hear 18:1-11. CrossRef

Rhode W, Smith P (1985) Characteristics of tone-pip response patterns in relationship to spontaneous rate in cat auditory nerve fibers. Hear Res 114:159-168.

Rhode W, Geisler CD, Kennedy DT (1978) Auditory nerve fiber response to wide-band noise and tone combinations. J Neurophysiol 41:692-704. Medline

Schaalje BG, Mcbride JB, Fellingham GW (2002) Adequacy of approximations to distributions of test statistics in complex mixed linear models. J Agricult Biol Environ Stats 7:512-524. CrossRef

Schaette R, McAlpine D (2011) Tinnitus with a normal audiogram: physiological evidence for hidden hearing loss and computational model. J Neurosci 31:13452-13457. CrossRef Medline

Schmiedt RA, Mills JH, Boettcher FA (1996) Age-related loss of activity of auditory-nerve fibers. J Neurophysiol 76:2799-2803. Medline

Sergeyenko Y, Lall K, Liberman MC, Kujawa SG (2013) Age-related cochlear synaptopathy: an early-onset contributor to auditory functional decline. J Neurosci 33:13686-13694. CrossRef Medline

Shaheen LA, Valero MD, Liberman MC (2015) Towards a diagnosis of cochlear neuropathy with envelope following responses. J Assoc Res Otolaryngol 16:727-745. CrossRef Medline

Shera CA (2004) Mechanisms of mammalian otoacoustic emission and their implications for the clinical utility of otoacoustic emissions. Ear Hear 25:86-97. CrossRef Medline

Simon H, Frisina RD, Walton JP (2004) Age reduces response latency of mouse inferior colliculus neurons to AM sounds. J Acoust Soc Am 116: 469-477. CrossRef Medline

Stamper GC, Johnson TA (2015) Auditory function in normal-hearing, noise-exposed human ears. Ear Hear 36:172-184. CrossRef Medline

Young ED, Barta PE (1986) Rate responses of auditory nerve fibers to tones in noise near masked threshold. J Acoust Soc Am 79:426-442. CrossRef Medline 